\title{
Cardiovascular suffering in childhood obesity
}

\author{
Georgiana Russu', Otilia-Elena Frasinariu², Laura Trandafir ${ }^{3}$ \\ ${ }^{1}$ Pediatric Cardiology Clinic, "Sf. Maria” Emergency Children's Hospital, lasi, Romania \\ ${ }^{2}$ Pediatric Clinic I, "Gr. T. Popa" University of Medicine and Pharmacy, lasi, Romania \\ ${ }^{3}$ Pediatric Clinic III, "Gr. T. Popa" University of Medicine and Pharmacy, Iasi, Romania
}

\begin{abstract}
Obesity-related cardiovascular disease is becoming more prevalent in conjunction with the rise in childhood obesity. Children with obesity may exhibit early signs of cardiovascular dysfunction: increased arterial stiffness, early atherosclerosis, changes in the myocardial structure and function. These are the result of their excess adiposity, often independent of other obesity-related comorbidities such as dyslipidemia and insulin resistance. Obesity in childhood predisposes to an increased risk of morbidity and cardiovascular mortality in adult. This review is to highlight the importance and need of programs for early detection, diagnosis, treatment and prevention of childhood obesity in order to decrease the incidence of the cardiovascular pathology in adults.
\end{abstract}

Keywords: obesity, children, atherosclerosis, myocardial function

\section{INTRODUCTION}

Obesity in children is defined as a body mass index (BMI) at or above the $95^{\text {th }}$ percentile for age and sex. A recent report from the National Health and Nutrition Examination Survey (NHANES) describes a dramatic increase in prevalence of childhood obesity in the United States from $7 \%$ to $18 \%$ in the last 30 years in all the age groups (1). Obesity also is strongly associated with other components of the cardiometabolic syndrome in adults (diabetes mellitus, hypertension, hyperlipidemia, atherosclerosis). Recognizing the importance of obesity and its related cardiovascular implications, the American Heart Association recently identified obesity as an independent and important modifiable risk factor for cardiovascular disease (2). A review of literature by Serdul et al revealed that the risk of adult obesity was at least twice as high for obese children as for non-obese children. About a third of obese preschool children were obese as adults, and about half of obese school-age children were obese as adults. The risk of adult obesity was greater for children who were at higher levels of obesity and for children who were obese at older ages (3). Adults who have been obese as children may have an even greater prevalence of risk factors for CVD, including hypertension and dyslipidemia, compared with those who had normal weight as children. It has also been shown that childhood obesity and central adiposity increased the risk for the metabolic syndrome in adulthood (4).

\section{Are We Programming Children to Be Obese?}

The ascendent slopes of some diseases incidence, while the genetic status of the populations is stable, were recently explained by the intervention of some epigenetic regulations, environmental dependent, which attend on the biologic evolution from the embrionary period to death (5). It was established a catalogue (still perfectible) of complex diseases encountered in adults but with fetal determination, with proved genetic transmition, or acquired by epigenetic resetations (obesity, atherosclerosis, type 2 diabetes mellitus etc).

Under environmental influences the genes function suffer reversible changes without changing of the DNA sequences by: DNA metilation, physical changes in the chromatin structure, and the action of some non-codant RNA molecules. The result is a genic modulation with impact on the phenotype of 
some diseases, as a response reaction to environmental factors (epigenetic risk factors): toxics, nutritional factors, infections, oncogenic factors, stress.

The epigenetic changes can be transmitted by gametes and are trans-generationals, demonstrated by the presence of obesity and type 2 diabetes mellitus in many generations. Also, it was proved a positive correlation between the mortality by diabetes and cardiovascular diseases with the nutritional status of the parents and grand-parents (6).

Boekelheide et al. (2012) studied in adults, after 50 years, the effects of starvation of the pregnant women from Holland during the Second World War and discovered that starving in the first trimester is correlated with glucose intolerance, cardiovascular diseases, arterial hypertension, dyslipidemia, obesity and affective disorders. Starvation during the second trimester correlated with glucose intolerance, pulmonary and renal diseases, and during the third trimester determined glucose intolerance and cardiovascular diseases (7).

Nutritional intake and maternal metabolic balance reflects on the birth weight, clasically considered as marker of risk for obesity in the child and future adult.

Many studies have shown that poor intrauterine nutrition, reflected on the low birth weight, newborns small for date ("economic phenotype") can develop even from adolescence adult metabolic diseases. Excluding the foetuses with low birth weight through hereditary or infectious pathology, it has reported increased prevalence of metabolic syndrome, coronary artery disease, arterial hypertension, dyslipidemia, insulin resistance (8).

In children with low weight by prematurity (weight corresponding to the gestational age) the umbilical cord plasma insulin concentrations were higher and persistent than those of full-term newborns (9).

High birth weight, especially if it is associated with maternal gestational diabetes correlates with elevated plasma insulin, obesity and metabolic syndrome in childhood and adulthood (10).

Obesity of the pregnant woman is accompanied by gestational complications: fetal macrosomy, gestational diabetes, preeclampsia. Bariatric surgery applied to obese women significantly reduces the risk of diabetes and hypertension in fetus (11).

Intrauterine environment modified by maternal obesity influence fetal development program, predisposing the teen and future adult to cardiovascular diseases, obesity and accelerated atherosclerosis.
Disturbed uterine environment and fetal malnutrition (by excess or deficit) together with other epigenetic risk factors disrupt the cardiovascular and metabolic gene commands, sometimes irreversible, reflecting along the life by the development of metabolic syndrome, obesity (sometimes with early onset), hypertension, dyslipidemia, hiperinsulinism, included in the category of fetal scheduled diseases (12).

Exaggerated weight gain from birth until at 18 months is associated with overweight or obesity later, localized adiposity, hypertension and elevated C-reactive protein levels, causing exaggerated thickness of the carotid wall measured at the age of 8 years $(13,14)$.

Breast-feeding is a key condition in the prevention of cardiovascular risk, obesity and numerous metabolic disturbances. WHO metaanalise (2013) on 75 observational studies has demonstrated the positive effect of breast-feeding in preventing obesity and hypertension of the adult (15).

In the same direction is the study on 822 Dutch youth (18-29 years) which showed that exclusive feeding with breast milk had a significant protective effect of body and visceral fat mass (BMI, abdominal perimeter and index abdominal perimeter hip circumference). Furthermore, it has demonstrated a significant positive correlation between the natural nutrition of premature babies and normal lipid profile at a later date (16).

\section{Vascular remodeling}

Obesity is recognized as independent predictor and major, but modifiable, risk factor for ATS and acute coronary heart disease. Obesity acts on the blood vessels by generalized endothelial dysfunction, accelerates the proliferation, migration and arterial smooth muscle cell hypertrophy and the recruitment of macrophages in the vascular wall. Generalized endothelial dysfunction and subsequent vasodilatation are regarded as the initial step in the pathogenesis of ATS, followed by stocking of low-density cholesterol (LDL). Dyslipidemia associated with obesity, insulin resistance, adipocitokines released by the adipose tissue accelerate further the process of ATS (17).

It was reported increased value of carotid intima media thickness (IMT) assessed by Doppler vascular ultrasound in obeses children and adolescents compared to children of the same age with normal BMI (18). The concept of vascular age was imposed through clinical use of this parameter for IMT evaluation on the percentiles scale for adult population of the same breed and sex (19). 
After evaluation of 70 children, aged 6-19 years, with obesity and risk factors for ATS (dyslipidemia, hypertension, insulin resistance, and exposure to tobacco smoke), Le and collab. have reported $75 \%$ cases with vascular age similar to a 45 -year-old adult (20).

Arterial stiffness is associated with increased cardiovascular risk in adult. A vascular system with normal elasticity request less work from the heart, increases coronary artery perfusion and is associated with a reduction in the rate of ATS production. Arterial compliance rises with age of the child, since arteries are getting bigger with growth and development. In children with obesity occur changes of some biophysical parameters of the aorta, reflecting the decreased elasticity of this artery (21).

In addition, it has been proven the effect of obesity on the properties of pulmonary artery wall, decreased elasticity and increased stiffness participating to the occurrence and progression of pulmonary hypertension and can be identified prior to the occurrence of clinical signs (22).

Autonomic nervous system dysfunction associated with obesity in children reduces the vagal variability of the heart rate (23), and also the baroreceptors sensitivity, with essential role in regulating blood pressure (TA) (24).

Hypertension and the stage of pre-hypertension are frequent and early complications in children with obesity, the frequency increasing in the last decade to $4 \%$ and $10 \%$ respectively (25). Hypertension in children is an important predictor for adult hypertension, but it is underdiagnosed. Identification and treatment of hypertensive youth encounters many difficulties because BP values range from one time to another depending on many physiological parameters and factors in the environment. Therefore, BP determination in outpatient assessment allows a real evaluation, allows risk stratification and prediction of cardiovascular prognosis. White coat hypertension, very common among children $(40 \%)$, is an important source of diagnostic error (26).

Three pathophysiological mechanisms were identified explaining the occurrence of hypertension in obese children: autonomic nervous system dysfunction, insulin resistance and alteration of the structure and function of vessels. Hyperactivity of the sympathetic autonomic nervous system has as a consequence cardiovascular manifestations, an increase in plasma catecholamine levels and increased peripheral sympathetic nervous system activity, clinically reflected by increased BP and heart rate at rest (27).
Insulin resistance in obese child was involved in the pathogenesis of hypertension, many studies reporting a positive correlation between serum insulin level and BP values at rest (28).

It has been suggested that obesity associated insulin resistance can prevent the insulin induced glucose consumption, but retaining the effect on sodium retention, which causes chronic volume overload and maintaining the BP at high values. Structural alteration of the vascular wall and increased arterial stiffness is another pathogenetic loop of HBP from obesity. Kortelainen and collaborators reported the increased weight of the heart and the presence of fat and calcium deposits in the coronaries wall at 210 necropsies carried out at obese children between the ages of 5-10 years old who had suffered violent death (29).

Adipose tissue in obesity is composed of mature adipocytes, preadipocytes, endothelial cells, and macrophages and presents specific features. Physiologically, mature adipocytes are active endocrine and paracrine cells, secreting a great number of mediators involved in various metabolic processes.

The best known is leptin, the hormone that acts as a regulating feedback factor which suppresses the hunger sensation at hypothalamic level. In the obese people, the circulating leptin is increased, in conjunction with adiposity. It was demonstrated the link between leptin and HB: leptin adjust the activity of the Na, K-ATPase modifying the renal sodium exchange, activates the renin-angiotensine-aldosterone axis and the sympathetic nervous system, appears to be linked to insulin resistance and acts together with other proinflammatory cytokines, inducing vascular oxidative stress and HBP (30).

Excessive adipose tissue is composed of hypertrophied adipocytes and macrophages and becomes dysfunctional, secreting proinflammatory adipokine cells: interleukin-6, $\alpha$ tumor necrosis factor, the plasminogen activating inhibitor and $\mathrm{C}$-reactive protein. Adiponectin, protein secreted in abundance by the adipose tissue, is an important stimulator of nitric oxide synthase activity and confers protection against oxidative stress and insulin resistance. Circulating levels of adiponectin are decreased in obesity, because the production is suppressed by proinflammatory adipokines. Taken together, these phenomena modifies glucose and lipids metabolism, causing vascular endothelial dysfunction and accelerating processes of atherosclerosis at the level of the vascular wall, thus increasing the cardiometabolic risk (31).

The kidney is the cause and also the victim of HBP. In the presence of insulin resistance, dyslipid- 
emia and glucose metabolism disturbances, favoring of HBP, may contribute to the kidney damage by the gradual loss of nephrons. Structural and functional alteration of the nephrons contributes to the BP increase. In pre-hypertensive children was demonstrated a reduction in glomerular filtration, associated with volume overload and proteinuria, suggesting that also the slightly elevated values of BP pose a risk of kidney impairment (32).

The treatment of pre-hypertension and stage 1 hypertension in obese children consists of lifestyle changes and reducing body weight. The drugs are recommended only starting with stage 2 hypertension (33). The beneficial effect of the decrease in body weight to reduce BP values was demonstrated. Thus, a meta-analysis of integrative studies regarding obesity and prevention of its complications has shown the effectiveness of the measures aimed at both diet and physical exercise to reduce BMI and normalize BP (34).

\section{Sleep apnea and obesity}

Obesity and overweight are known risk factors for sleep apnea, which contribute to the occurrence of cardiovascular complications through various pathophysiological mechanisms. Recurrent episodes of partial or total obstruction of the airway during sleep, having as consequence hypoxia and hypercapnia, causes neuroumoral changes, oxidative stress, inflammatory response and metabolic dysfunction. Thus, activation of the sympathetic nervous system and endothelial dysfunction caused by the inflammatory response from blood vessels induced by the release of pro-inflammatory mediators (C-reactive protein, endothelin-1) determines the occurrence of HBP. Other mechanisms activated by sleep apnea in obesity are: alteration of arterial baroreflex activity, kidney dysfunction, hyperleptinemia, resistance to insulin, renin-angiotensin system and oxidative stress activation (35). The frequent hypoxic episodes determine repeating episodes of pulmonary vasoconstriction which have as consequence pulmonary hypertension (36).

\section{Cardiac suffering}

Obesity is associated with increased metabolic demands caused by increased quantity of adipose tissue; increased preload growth determined by an increase in the blood volume is associated with increased afterload determined by arterial stiffness and resistance. Consequently, eccentric and concentric hypertrophy occurs in the left ventricle (VS), correlated with the degree and duration of as- sociation with obesity and HBP (37). It is proven that the dimensions of the left atrium and left ventricle (LV), and also the LV mass are bigger in obese children than in children with normal BMI.

Epicardic fat tissue stored around the heart between pericardium and the outer wall of the myocardium is proposed as a cardiovascular risk factor (38). Echocardiographic assessement of epicardic adipose tissue showed a positive correlation with visceral adipose tissue depots. There is a small amount of adipose epicardic tissue in subjects with normal BMI, but the limit value considered normal has not yet been established. However, a recent study has suggested the figure of $4.1 \mathrm{~mm}$ as reference value in children (39).

Epicardic adipose deposit size correlates with insulin resistance, coronary artery disease, carotid wall thickness and arterial stiffness in adult. Measurement of epicardic tissue may be useful for assessing the cardiovascular risk in children.

Macroscopic changes of the heart in obese children are: increased cardiac weight, hypertrophy of $\mathrm{LV}, \mathrm{RV}$, and ventricular dilation. Microscopically, it is observed myocytes hypertrophy and myocardial fibrosis, followed by apoptosis and necrosis of the myocytes, capillaries rarefaction and inflammatory infiltrate (40).

The concept of lipotoxicity in obese cardiomyopathy is based on experimental discovery of intra and intercellular fat deposit, causing degradation of myocytes, apoptosis, atrophy and heart dysfunction (41). Similarly, studies in humans have shown increased content of triglyceride at myocardial level (42).

The first change of heart function that appears in obesity is diastolic dysfunction, in the form of disturbed relaxation and/or compliance of the myocardium. It occurs independently or in combination with other changes characteristic to the obese cardiomyopathy, such as LV hypertrophy. Moreover, the severity of the diastolic dysfunction is directly proportional to the duration of obesity (43).

LV hypertrophy determines also subendocardial ischemia, a phenomenon accentuated by the presence of epicardial fat which, by his size, restricts the normal mechanics of heart (44).

Gradually, systolic dysfunction installs correlated with the severity of obesity, as demonstrated by the modern techniques of echocardiography (45).

Visceral adiposity is independently associated with the risk of cardiovascular disease. Fatty tissue is more and more acknowledged as a metabolically active organ. When the storage capacity of fat tis- 
sue achieve the maximum, excess fat can be stored in the ectopic tissue, such as skeletal muscle, heart, vessel, and kidney, causing an $\mathrm{f}$ insulin-resistant status. This results in lipolysis with excessive release of free fatty acids and other peptides derived from adipocytes, such as leptin, rezistine, $\alpha$ tumor necrosis factor, adiponectin, plasminogen activation inhibitor and interleukin 6 (46).

Adipose tissue is infiltrated with macrophages. Most inflammatory cytokines released from adipose tissue are derived from macrophages which infiltrate functional dysfunctional adipose tissue. Cytokines environment maintains the macrophages recruitment. Cancello and collab have shown improvement of the inflammatory status by reducing macrophage infiltration from the adipose tissue and improvement of insulin sensitivity in obese subjects who have lost weight (47).

\section{Cardiovascular effect of obesity treatment}

Current guidelines recommend lifestyle changes, diet, reducing sedentary activities and physical exercises program for prevention and treatment of childhood obesity. BMI is used as a marker of treatment success. More aggressive measures, such as drug treatment and bariatric surgery are reserved for the adolescents with severe obesity who have failed conventional therapeutic measures (48). Physical exercise has a favourable effect on BMI and adiposity dimensions in short term (10 weeks) and medium term (4 months). Many studies have demonstrated improvement in cardiac and vascular function after 6 months of dietary measures and physical exercise, with significant improvement of diastolic dysfunction (49). In adolescents with morbid obesity, bariatric surgery improved cardiac geometry, corrected diastolic function and decreased epicardial fat; the assessment was made after $10 \pm 3$ months postoperatively (50). Huang et al have noted an improvement in the status of adhesion molecules (selectine E), parameters of fibrinolysis (the plasminogen activation inhibitor), in the amount of triglycerides and glucose homeostasis in patients who have obtained a reduction in BMI after six months of life style modification (51). These improvements have been accompanied by improving cardiometabolic risk factors, adiposity and biomarkers.

\section{CONCLUSIONS}

Obesity, fetal scheduled disease, with onset anytime along childhood under the influence of a context of risk factors, is accompanied (in proportion to the severity) by cardiovascular impairment. The child and adolescent obesity is associated with cardiovascular risk on short and medium term, which includes the hemodynamic changes and structural and functional changes at the level of the heart and vessels. Arterial stiffness and relaxation proprieties of the ventricular myocardium is physiologically changing with increasing age. Obesity in childhood accelerates these physiological processes, increasing the risk of cardiovascular disease in adulthood, but determines cardiovascular damage also in the child. Programs of prevention, diagnosis and treatment of obesity in children and adolescents should include early identification of cardiovascular dysfunction and follow-up as correcting BMI. Young age ensure reversibility of cardiovascular side effects of obesity, only with early inclusion of the patient in the recovery program.

\section{Conflict of interest: none declared} Financial support: none declared

\section{REFERENCES}

1. Barlow S.E. Expert committee recommendations regarding the prevention, assessment and treatment of child and adult overweight and obesity: summary report, Pediatrics 2007; 120 suppl 4: 164-92.

2. Eckel R.H., Kahn R. et al. Preventing cardiovascular disease and diabetes: a call to action from the American Diabetes Association and the American Heart Association, Circulation 2006, 113: 2943-2946.

3. Serdula M.K., Ivery D., Coates R.J. et al. Do obese children become obese adults? A review of the literature. Prev Med 1993, 22: 167-77.

4. Bridger T. Childhood obesity and cardiovascular disease, Paediatr Child Health 2009, 14 (3): 177-182.

5. Smith R., Mill J. Epigenetics and chronic diseases: an overview, in Roach H. et al. (eds.): Epigenetic aspects of chronic diseases. Springer-Verlag 2011.

6. Rockholm B., Baker J., Sorensen T. The levelling of the obesity epidemic since the year 1999-a review of evidence and perspectives. Obes. Rev. 2010, 11, 12: 835-846.

7. Boekelheide citat de Buzinschi S. De ce este importantă epigenetica pentru pediatrie? Revista Română de Pediatrie 2016, LXV, 3:285-291.
8. Bridger T. Childhod obesity and cardiovascular disease, Paediatr Child Health 2009; 14(3): 177-182.

9. Radovick S. et al. Preterm birt hand random plasma insulin at birt hand in early childhood. JAMA 2014, 311, 6:587-596.

10. Nadeau K.J., Maahs D.M., Daniels S.R., Eckel R.H. Childhood obesity and cardiovascular disease: links and prevention strategies, Nat Rev Cardiol 2015, 8(9): 513-525.

11. Stone R.A., Huhman J., Istvan N. et al. Pregnancy outcomes following after bariatric surgery. Journal of women's health. 2011, 20, 9:1363-1366.

12. Barker D.J.P. Fetal origins of coronary heart disease, BMJ 1995, 311: 171-174.

13. Skilton M.R., Marks G.B., Ayer J.G. et al. Weight gain in infancy and vascular risk factors in later childhood, Pediatrics 2013, 131: e1821-1828.

14. Skilton M.R., Sullivan T.R., Ayer J.G. et al. Weight gain in infancy is associated with carotid extra-medial thickness in later childhood, Atherosclerosis 2014, 233: 370-374. 
15. World Health Organization. Editors: B.L. Horta; C.G. Victora. Long-term effects of breastfeeding: a systematic review 2013.

16. De Kroon M., Renders C., Buskermolen M., Van Wouwe J., van Buuren S., Hirasing R. The Terneuzen Birth Cohort. Longer exclusive breastfeeding duration is associated with leaner body mass and a healthier diet in young adulthood. BMC Pediatr 2011; 11(1):33.

17. Lastra G., Manrique C., Whaley-Connel A. Hypertension and the cardiometabolic syndrome, J Clin Hypertens 2005, 7: 471-476.

18. Cote A.T., Harris K.C. et al. Childhood obesity and cardiovascular dysfunction, JACC 2013, 62 (15):1309-1319.

19. Stein J.H. Carotid intima-media thickness and vascular age: you are only as old as your arteries look, J AmSoc Echocardiogr 2004, 17: 686-689.

20. Le J., Zhang D., Mences S. et al. Vascular age is advanced in children with atherosclerosis-promoting risk factors, Circ Cardiovasc Imaging 2010, 3: 8-14.

21. Pac F.A., Guray Y., Polat T.B. Wall motion velocities of ascending aorta measured by tissue Doppler imaging in obese children, Pediatr Int 2010, 52: 778-784.

22. Sanz J., Kariisa M. et al. Evaluation of pulmonary artery stiffness in pulmonary hypertension with cardiac magnetic resonance, J Am Coll Cardiol Img 2009, 2:286-295.

23. Vanderlei L.C., Pastre C.M. et al. Analysis of cardiac autonomic modulation in obese and eutrophic children, Clinics (Sao Paolo) 2010, 65: 789-792.

24. Lazarova Z., Tonhajzerova I. et al. Baroreflex sensitivity is reduced in obese normotensive children and adolescents, Can J Physiol Pharmacol 2009, 87: 565-571.

25. Jain S., Bhatt: Childhood obesity and hypertension, $J$ Childh Obes 2016, 1 (2): 8.

26. Urbina E., Alpert B., Flynn J., Hayman L., Harshfield G., et al. Ambulatory blood pressure monitoring in children and adolescents: Recommendations for standard assessment. A scientific statement from the american heart association atherosclerosis, hypertension, and obesity in the young committee of the council on cardiovascular disease in the young and the council for high blood pressure research, Hypertension 2008, 52: 433-451.

27. Sorof J., Daniels S. Obesity hypertension in children, Hypertension 2002, 40: 441-447.

28. Kanai H., Matsuzawa Y., Keno Y. et al. Hypertension in obese children: fasting serum insulin levels are closely correlated with blood pressure, Int J Obes 1990, 14: 1047-1056.

29. Nguyen T., Lau D.C.W. The obesity and its impact on hypertension, Can J Cardiol 2012, 28: 326-333.

30. Leiter L.A., Fitchett D.D., Gilbert R.E. et al. Cardiometabolic risk in Canada: a detailed analysis and position paper by the cardiometabolic risk working group, Can J Cardiol 2011, 27: e1-33.

31. Bakris G.L., Ritz E. The message for World Kidney Day 2009: hypertension and kidney disease - a marriage that should be prevented, Pediatr Nephrol 2009:24: 427-430.

32. Jain S., Bhatt G.C. Childhood obesity and hypertension, J Child Obes 2016, 1 (2): 8-9.

33. Cai L., Wu Y., Wilson R.F. et al. Effect of childhood obesity prevention programs on blood pressure: A systematic review and meta-analysis, Circulation 2014, 129: 1832-1839.
34. Dorresteijn J.A., Visseren F.L., Spiering W. Mechanisms linking obesity to hypertension, Obes Rev 2012, 13: 17-26.

35. Ho T.F. Cardiovascular risk associated with obesity in children and adolescents, Ann Acad Med Singapore 2009, 38: 48-56.

36. Vasan R.S. Cardiac function and obesity, Heart 2003, 89: 1127-1129.

37. lacobellis G., Assael F., Ribaudo M.A. et al. Echocardiographic epicardial adipose tissue is related to anthropometric and clinical parameters of metabolic syndrome: a new indicator of cardiovascular risk, J Clin Endocrinol Metab 2003, 88: 5163-5168.

38. Abaci A., Tascilar M.E., Saritas T. et al. Threshold value of subepicardial adipose tissue to detect insulin resistance in obese children, Int J Obes 2009, 33: 440-446.

39. Wong C., Marwick T.H. Obesity cardiomyopathy: pathogenesis and pathophysiology, Nat Clin Pract Cardiovasc Med 2007, 4: 436-443.

40. Chiu H.C., Kovacs A., Ford D.A. A novel mouse model of lipotoxic cardiomyopathy, J Clin Invest 2001, 107: 813-822.

41. Szczepaniak L.S., Dobbins R.L., Metzger G.J. Myocardial triglycerides and systolic function in humans: in vivo evaluation by localized proton spectroscopy and cardiac imaging, Magn Reson Med 2003, 49: 417-423.

42. Wong C.Y., O'Moore-Sullivan T., Leano R. Alterations of left ventricular myocardial characteristics associated with obesity, Circulation 2004, 101: 3081-3087.

43. De Pergola G., Nardecchia A., Giagulli V.A. et al. Obesity and heart failure, Endocr Metab Immune Disord Drug Targets 2013, 13 (1): 51-57.

44. Border W.L., Michelfelder E.C., Glascock B.J. et al. Color M-mode and Doppler tissue evaluation of diastolic function in children: simultaneous correlation with invasive indices, J Am Soc Echocardiogr 2003, 16: 988-994.

45. Montani J.P., Carroll J.F., Dwyer T.M. et al. Ectopic fat storage in heart, blood vessels and kidneys in the pathogenesis of cardiovascular diseases, Int J Obes Relat Metab Disord 2004, 28 (4): 558-565.

46. Cancello R., Henegar C. et al. Reduction of macrophage infiltration and chemoattractant gene expresion changes in white adipose tissue of morbidly obese subjects after surgery-induced weight loss, Diabetes 2005, 54: 2277-2286.

47. Kirk S., Scott B.J., Daniels S.R. Pediatric obesity epidemic: treatment options, J Am Diet Assoc 2005, 105:S44-51.

48. Cote A.T., Harris K.C. et al. Childhood obesity and cardiovascular dysfunction, JACC 2013, 62 (15):1309-1319.

49. Gaborit B. Jacquier A., Kober F. et al. Effects of bariatric surgery on cardiac ectopic fat: lesser decrease in epicardial fat compared to visceral fat loss and no change in myocardial triglyceride content, J Am Coll Cardiol 2012, 60: 1381-1389.

50. Huang F., del-Rio-Navarro B.E. et al. Weight loss induced by 6-month lifestyle intervention improves early endothelial activations and fibrinolysis in obese adolescents, Child Care Health Dev 2011, 37: 377-384. 